\title{
Estimating Prevalence, Demographics, and Costs of ME/CFS Using Large Scale Medical Claims Data and Machine Learning
}

\author{
Ashley R. Valdez ${ }^{1}$, Elizabeth E. Hancock ${ }^{1}$, Seyi Adebayo ${ }^{1}$, David J. Kiernicki ${ }^{1}$, \\ Daniel Proskauer ${ }^{2}$, John R. Attewell ${ }^{3}$, Lucinda Bateman ${ }^{4}$, Alfred DeMaria Jr. ${ }^{5}$, \\ Charles W. Lapp ${ }^{6}$, Peter C. Rowe ${ }^{7}$ and Charmian Proskauer ${ }^{8 *}$
}

${ }^{1}$ Optum Enterprise Analytics, UnitedHealth Group, Minneapolis, MN, United States, ${ }^{2}$ Optum Technology, UnitedHealth Group, Minneapolis, MN, United States, ${ }^{3}$ Innovation, Research, and Development, UnitedHealth Group, Minneapolis, MN, United States, ${ }^{4}$ Bateman Horne Center, Salt Lake City, UT, United States, ${ }^{5}$ Bureau of Infectious Disease and Laboratory Sciences, Massachusetts Department of Public Health, Boston, MA, United States, ${ }^{6}$ Hunter-Hopkins Center, Charlotte, NC, United States, ${ }^{7}$ Children's Center Chronic Fatigue Clinic, Johns Hopkins University School of Medicine, Baltimore, MD, United States, ${ }^{8}$ Massachusetts ME/CFS \& FM Association, Quincy, MA, United States

Techniques of data mining and machine learning were applied to a large database of medical and facility claims from commercially insured patients to determine the prevalence, gender demographics, and costs for individuals with provider-assigned diagnosis codes for myalgic encephalomyelitis (ME) or chronic fatigue syndrome (CFS). The frequency of diagnosis was 519-1,038/100,000 with the relative risk of females being diagnosed with ME or CFS compared to males 1.238 and 1.178, respectively. While the percentage of women diagnosed with ME/CFS is higher than the percentage of men, ME/CFS is not a "women's disease." Thirty-five to forty percent of diagnosed patients are men. Extrapolating from this frequency of diagnosis and based on the estimated 2017 population of the United States, a rough estimate for the number of patients who may be diagnosed with ME or CFS in the U.S. is 1.7 million to 3.38 million. Patients diagnosed with CFS appear to represent a more heterogeneous group than those diagnosed with ME. A machine learning model based on characteristics of individuals diagnosed with ME was developed and applied, resulting in a predicted prevalence of 857/100,000 $(p>0.01)$, or roughly 2.8 million in the U.S. Average annual costs for individuals with a diagnosis of ME or CFS were compared with those for lupus (all categories) and multiple sclerosis (MS), and found to be 50\% higher for ME and CFS than for lupus or MS, and three to four times higher than for the general insured population. A separate aspect of the study attempted to determine if a diagnosis of ME or CFS could be predicted based on symptom codes in the insurance claims records. Due to the absence of specific codes for some core symptoms, we were unable to validate that the information in insurance claims records is sufficient to identify diagnosed patients or suggest that a diagnosis of ME or CFS should be considered based solely on looking for presence of those symptoms. These results show that a prevalence rate of $857 / 100,000$ for ME/CFS is not unreasonable; therefore, it is not a rare disease, but in fact a relatively common one.

Keywords: ME/CFS, myalgic encephalomyelitis, chronic fatigue syndrome, prevalence, costs, machine learning, data mining 


\section{INTRODUCTION}

Myalgic Encephalomyelitis (ME) and Chronic Fatigue Syndrome (CFS) are serious, debilitating conditions that impose a burden of illness on millions of people in the United States and around the world (1).

Multiple case definitions have been used to define ME and CFS. Those for ME require the presence of post-exertional malaise and tend to identify a more severely ill subset of the broader ME and CFS population (2). Although there are separate diagnostic codes for ME and CFS, the descriptions in the International Classification of Diseases (ICD) ${ }^{1}$ listings are the same. The two terms ME and CFS have been conflated, and as of 2016, U.S. federal health agencies have used the combined term $\mathrm{ME} / \mathrm{CFS}$ to refer to this disease.

$\mathrm{ME} / \mathrm{CFS}$ is an acquired, chronic, multi-systemic disease characterized by significant relapse after physical, cognitive, or emotional exertion of any sort. The disease includes immune, neurological and cognitive impairment, sleep abnormalities, and autonomic dysfunction, resulting in significant functional impairment accompanied by a pathological level of fatigue. The cause of the disease remains unknown, although in many cases symptoms may have been triggered by an infection or other prodromal event [U.S. Department of Health and Human Services, (3)].

The underlying etiology is not known. There is no readily available laboratory test to diagnose ME/CFS, no FDA-approved drug for ME/CFS, and no cure. Many ME/CFS patients experience significant disability. At least one-quarter of ME/CFS patients are house- or bedbound at some point in their lives $(4,5)$. The direct and indirect economic costs of ME/CFS to society have been estimated at $\$ 17$ to $\$ 24$ billion annually (6), including $\$ 9.1$ billion attributed to lost household and labor force productivity (7).

Assigning a diagnosis of ME/CFS in the clinical setting often takes years. Many physicians are uninformed or misinformed about the disease (1). It has been estimated that $84-91 \%$ of patients affected by ME/CFS are not diagnosed with the disease (8).

Thus, improving diagnosis and optimizing management can have significant economic and public health consequences (2). Without good data on the prevalence of ME/CFS, it is difficult to allocate resources for research of all kinds (etiology, pathophysiology, treatment, etc.), as well as for medical education, that would be commensurate with the burden of the disease.

This study uses data from a large sample of the general population insured by a major commercial health insurance

\footnotetext{
${ }^{1}$ ICD codes for ME and CFS

Available online at: https://www.icd10data.com/ICD10CM/Codes/G00-G99/G89G99/G93-/G93.3

Available online at: https://www.icd10data.com/ICD10CM/Codes/R00-R99/R50R69/R53-/R53.82

Available online at: http://www.icd9data.com/2014/Volume1/320-389/320-327/ 323/323.9.htm

Available online at: http://www.icd9data.com/2014/Volume1/780-799/780-789/ 780/780.71.htm
}

carrier to look at characteristics of clinician-diagnosed $\mathrm{ME}$ and CFS patients. We applied techniques of data mining and machine learning to a large medical claims database to investigate the prevalence, characteristics, and costs for individuals with $\mathrm{ME} / \mathrm{CFS}$.

\section{METHODS}

This study examined de-identified physician and hospital data from a large claims processing database from Optum, a large healthcare information and services company, which allowed us to describe features of physician-diagnosed ME/CFS patients and to compare this group to the general insured population. The overall sequence of the study is shown in Figure 1.

\section{Data Sources}

The Optum database contains membership, provider, claims, and ancillary data on over 101 million former and current members. The database contains no identifying information on individuals (names, addresses, etc.), but each individual's claims data are linked. The database contains a primary diagnosis and up to four additional diagnostic codes for each claim.

The primary dataset used in this study includes medical and facility insurance claims for nearly 50 million $(49,963,500)$ individuals age 0 through 89 who had at least one medical or one facility claim. Only medical (e.g., from doctor's offices and including any tests or procedures that were conducted or ordered there) and facility (e.g., hospital) claims were part of the data sets used in this study. Prescription drug claims were not included.

The database captured all medical and facility claims, and did not require that the individual have continuous insurance coverage over a specific period of time. The data used in this study are primarily from individuals enrolled during the years 20112016, 2016 being the last year for which complete enrollment data were available. All claims for these individuals were considered including claims from the year 2017 if available.

Approximately $80 \%$ of individuals in this dataset were insured by commercial health insurance; close to $20 \%$ had coverage from Medicare, the U.S. government program for individuals age 65 and over and for certain individuals with disabilities. The dataset did not include any claims associated with Medicaid, the U.S. government program for low-income individuals. For some of the topics in this study, sub-sets of the dataset were used.

We used diagnosis codes which were assigned and entered into the patient's record. From the code alone we could not determine what case definition or clinical diagnostic criteria were used to make the diagnosis.

During the years 2011-2016 two different sets of ICD codes $^{2}$ were in use, ICD-9-CM and ICD-10-CM. Both versions included codes that were used for CFS (Chronic Fatigue Syndrome) and

\footnotetext{
${ }^{2}$ ICD codes related to fatigue

Available online at: http://www.icd9data.com/2014/Volume1/780-799/780-789/ 780/780.79.htm

Available online at: https://www.icd10data.com/ICD10CM/Codes/R00-R99/R50R69/R53-/R53.81

Available online at: https://www.icd10data.com/ICD10CM/Codes/R00-R99/R50R69/R53-/R53.83
} 


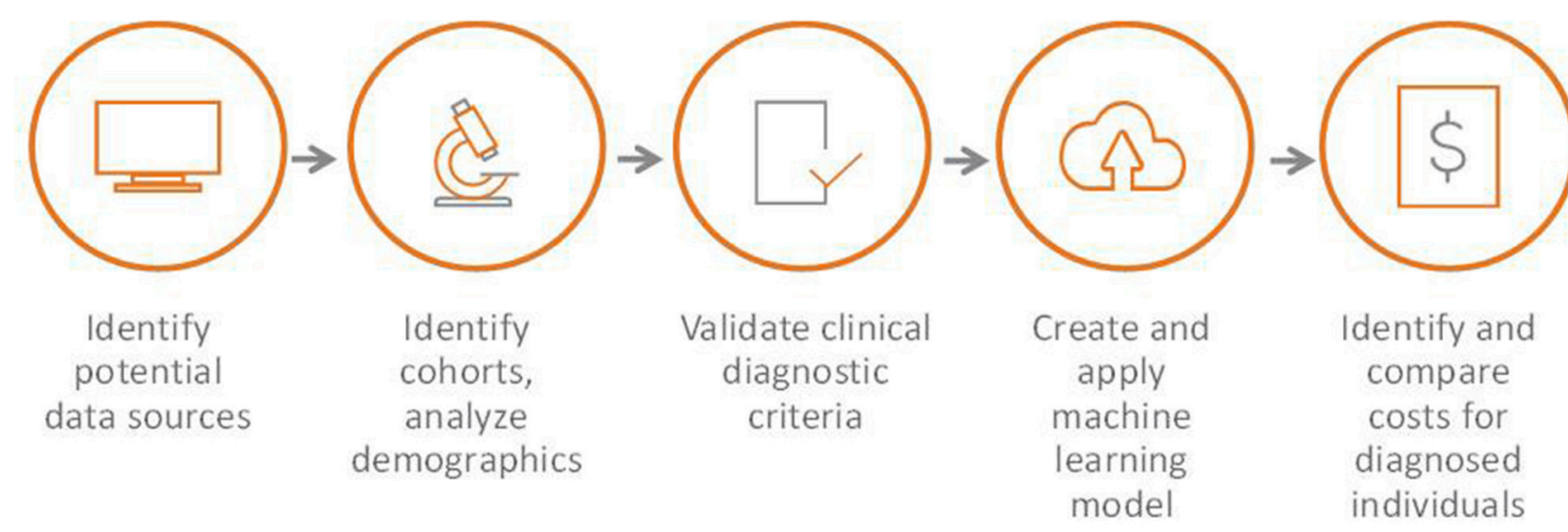

FIGURE 1 | Graphic showing sequence of steps in this study.

TABLE 1 | ICD codes used for diagnosis of ME and CFS.

\begin{tabular}{lcc}
\hline Diagnosis & $\begin{array}{c}\text { ICD-9-CM } \\
\text { (retired Oct 1, } \\
\text { 2015) }\end{array}$ & ICD-10-CM \\
\hline ME (Myalgic Encephalomyelitis) & 323.9 & G93.3 \\
CFS (Chronic Fatigue Syndrome) & 780.71 & R53.82
\end{tabular}

ME (Myalgic Encephalomyelitis) (Table 1). ME and CFS were analyzed separately in some analyses. The lack of specificity and interrelationships of these codes (see Appendix 1 in Supplementary Material, Interrelationships of ICD codes used for Chronic Fatigue Syndrome (CFS) and Myalgic Encephalomyelitis (ME) in the U.S. as of October, 2018) introduce a degree of uncertainty; however, these are the diagnostic codes used for $\mathrm{ME}$ and CFS, and provide the best available baseline for this type of study at this point in time.

\section{Frequency of ME/CFS Diagnosis and Demographics of Diagnosed Population}

Main dataset: The general population (all individuals in the database) and the diagnosed population (all those with codes for ME or CFS) were examined for distribution by current (as of 2017) age and gender. Queries were run against the entire data set and two subsets. Separate queries were run to eliminate duplications if an individual had codes for both ME and CFS.

The initial analysis was conducted using the entire dataset of 49,963,500 individuals who had at least one medical or one facility claim. Claims associated with these individuals were examined for presence of one of the four diagnosis codes as the primary diagnosis. Length of insurance enrollment was not considered for this group.

Subset 1: The first subset analysis examined individuals who were continuously enrolled in the same insurance plan for the entire 2011-2016 period, providing longer length of enrollment, and more complete medical history.
Subset 2: The second subset consisted of individuals who were continuously enrolled in the same insurance plan for between 2 and 4 years at any time between 2011 and 2016 . This group more accurately represents the central tendencies of the data and eliminates outliers. For this query we used codes from all 5 diagnosis code fields in the analysis, not just the primary diagnosis. All claims for these individuals were included. In addition to the base query, we also separately analyzed demographic data for individuals in this subset who were diagnosed with ME only, without including those diagnosed with CFS alone.

For all datasets, the reference population is all the individuals in that dataset.

\section{Validation of Clinical Diagnostic Criteria}

An important component of this study was an examination of symptom codes in medical records to determine if the diagnosis of ME/CFS could be confirmed by the presence of a unique cluster of symptoms, such as those in the diagnostic criteria proposed in the 2015 report from the National Academy of Medicine (1), which requires 4 or 5 core symptoms that were determined to be strongly supported by evidence as accurately identifying ME/CFS (Appendix 2 in Supplementary Material).

In order to maximize the probability of being able to identify symptoms, we limited analysis to the population continuously enrolled for the entire 2011-2016 period (subset 1), since longer enrollment provides a more complete history.

\section{Estimating Prevalence Using Machine Learning}

We applied the techniques of machine learning to predict the prevalence of ME using another method of analyzing our claims data. (For more information about machine learning, see Appendix 3 in Supplementary Material). From the cohort of individuals who were continuously enrolled for 2 to 4 years, individuals under the age of 15 years were removed in order to minimize features that would be created from predominantly pediatric care. To create the machine learning modeling cohort, 
TABLE 2 | ICD codes used for the diagnosis of multiple sclerosis and lupus.

\begin{tabular}{lcc}
\hline Diagnosis & $\begin{array}{c}\text { ICD-9-CM (retired } \\
\text { Oct 1, 2015) }\end{array}$ & ICD-10-CM \\
\hline MS (Multiple Sclerosis) & 340 & G35 \\
Lupus (includes all subcategories) & 710 & M32, L93 \\
\hline
\end{tabular}

we included all members of the diagnosed population as well as a random sample of $25 \%$ from the remaining general population.

The modeling cohort was randomly split into a training, validation, and testing set per data science protocol. The training set was rebalanced for modeling with a 50-50 random split, so that the diagnosed and general population were evenly split and the model could train on positive and negative classes evenly. The training set was used to train the model. The validation set was used to tune the model. The testing set was used to do a final test on the finished model.

The model was built using XGBoost, an open source implementation of the boosted tree method of supervised learning. The final model contained 507 features which included medical claim codes, age, and gender information. The validation set was used for prevalence estimates. Prevalence was estimated from individuals that the model predicted to have ME at a $99 \%$ probability and dividing by the total number in the dataset.

\section{Costs}

To analyze the financial impact of the disease, we used data from the main data set for the years 2012 through 2016. This time period was chosen because it includes the largest number of individuals and the most years of claims data. We focused on individuals with the ME diagnosis code because we speculated that assignment of the less well-known ME diagnosis code might better represent the characteristics and diagnostic criteria of $\mathrm{ME} / \mathrm{CFS}$, and therefore this would be a more specific group. We only considered individuals from the overall cohort who were 13 years of age or older, since the incidence of ME in young children is much smaller.

Costs used were the standard allowed payment (contracted rate) for all provider services which may have ultimately been paid by either the insurer or related patient responsibility associated with the claim such as patient co-payment or deductible, if any.

We looked at the yearly costs related to claims for individuals diagnosed with $\mathrm{ME}$ vs. all other individuals in the reference population. The average annual cost per individual was calculated on medical claims $>\$ 0$ for each year from 2012 to 2016. Costs were not adjusted for inflation. Costs included both those paid by the insurer at the standard allowed payment for all provider services and the related patient responsibility associated with the claim such as patient co-payment or deductible, if any. Only medical and facility claims were considered, and we did not analyze the content of claims that contributed to the costs.

To put the cost in context, we also looked in the same way at annual costs related to claims for two similar diseases which are often compared to ME/CFS, multiple sclerosis and lupus
TABLE 3 | Prevalence of ME diagnosis for varying lengths of continuous enrollment (any years).

\begin{tabular}{lccc}
\hline $\begin{array}{l}\text { Continuous years } \\
\text { enrolled }\end{array}$ & Diagnosed & $\begin{array}{c}\text { General } \\
\text { Pop. }\end{array}$ & $\begin{array}{c}\text { Prevalence per } \\
\mathbf{1 0 0 , 0 0 0}\end{array}$ \\
\hline $0-1$ & 2,668 & $3,648,421$ & 73 \\
$1-2$ & 11,070 & $13,422,797$ & 82 \\
$2-3$ & 7,883 & $7,339,562$ & 107 \\
$3-4$ & 6,337 & $4,438,630$ & 143 \\
$4-5$ & 6,375 & $3,660,868$ & 174 \\
$5-6$ & 2,971 & $1,670,694$ & 178 \\
$6-7$ & 4,925 & $2,629,342$ & 187 \\
Total & 42,229 & $36,810,314$ & 115 \\
\hline
\end{tabular}

erythematosus. ICD codes used in these queries are shown in Table 2.

\section{RESULTS}

\section{Prevalence of ME Diagnosis vs. Average Length of Enrollment}

In creating Subset 2, we compared the average length of continuous enrollment for individuals diagnosed with MEonly vs. the general insured population. The average length of enrollment of all individuals in the database is just over 2 years (980 days). Individuals with a diagnosis code for ME but not for CFS, on average, have been enrolled for just over 3 years $(1,204$ days). We therefore chose the length of continuous enrollment from 2 to 4 years to most accurately represent the central tendencies of the data and eliminate outliers for both the general and the ME-diagnosed population. Subset two includes individuals with a diagnosis of ME in any of the diagnosis fields in the claims (primary diagnosis plus up to four additional secondary diagnoses).

Table 3 shows the number of individuals continuously enrolled for periods of from 1 to 7 years who had a diagnosis code of ME (but not CFS). Note that as the length of continuous enrollment increases, the proportion with an ME diagnosis also increases, as would be expected as the opportunity for diagnosis is extended.

\section{Frequency of ME/CFS Diagnosis}

Tables 4, 5 show diagnostic codes for ME and CFS and prevalence of these diagnoses for the three population sets. Data columns for ME and CFS include all individuals who had that as the primary diagnosis code in any of their claims. Prevalence for each of the three groups was calculated by dividing the number of diagnosed individuals by the total in the reference population. Some individuals might have had both codes within their set of claims; separate queries were run to eliminate this duplication. Table 4 shows the prevalence for ME and CFS separately. Table 5 shows the prevalence for ME+CFS with and without duplication. Without duplication, prevalence of ME/CFS was 519/100,000 in the main dataset (non-continuous enrollment), 669/100,000 in Subset 1 (continuous enrollment for the entire period), and 
TABLE 4 | Summary of prevalence of ME and CFS in three studied cohorts.

\begin{tabular}{|c|c|c|c|c|c|c|c|c|}
\hline \multirow[t]{2}{*}{ Population } & \multicolumn{4}{|c|}{ ME } & \multicolumn{4}{|c|}{ CFS } \\
\hline & G93.3 & 323.9 & Total w/Dups & per $100 \mathrm{~K}$ & R53.82 & 780.71 & Total w/dups & per $100 \mathrm{~K}$ \\
\hline Main dataset & 16,305 & 9,263 & 25,568 & 51 & 140,947 & 99,929 & 240,876 & 482 \\
\hline Subset 1 & 1,044 & 1,030 & 2,074 & 81 & 6,635 & 10,234 & 16,869 & 661 \\
\hline Subset 2 & 10,196 & 3,945 & 14,141 & 121 & 87,282 & 57,614 & 144,896 & 1,236 \\
\hline
\end{tabular}

TABLE 5 | Summary of prevalence of ME + CFS in the three studied cohorts, and with duplicates eliminated.

\begin{tabular}{|c|c|c|c|c|c|}
\hline \multirow[t]{2}{*}{ Population } & \multicolumn{2}{|c|}{ ME+CFS } & \multicolumn{2}{|c|}{ Union ME+CFS } & \multirow{2}{*}{$\begin{array}{c}\text { Reference } \\
\text { Total }\end{array}$} \\
\hline & Total & per $100 K$ & Total no dups & per $100 \mathrm{~K}$ & \\
\hline Main dataset & 266,444 & 533 & 259,275 & 519 & 49,963,500 \\
\hline Subset 1 & 18,943 & 742 & 17,074 & 669 & $2,553,722$ \\
\hline Subset 2 & 159,037 & 1,357 & 121,632 & 1,038 & $11,720,401$ \\
\hline
\end{tabular}

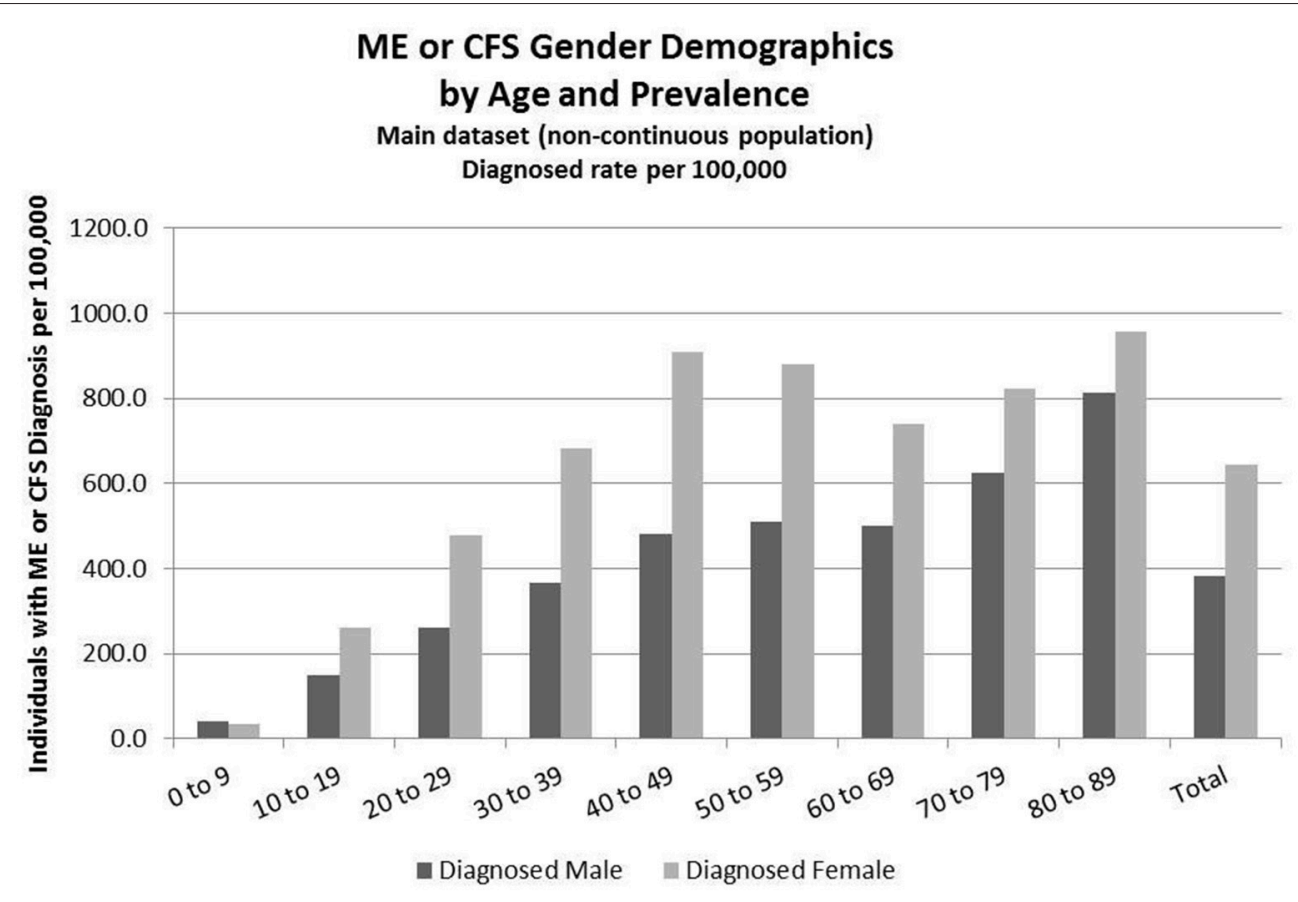

FIGURE 2 | ME or CFS gender demographics by age and prevalence (main dataset, non-continuous enrollment).

1,038/100,000 in Subset 2 (continuous enrollment for any 2-4 year period). For Subset 2 only, up to four secondary diagnosis fields were included from the claims in addition to the primary diagnosis.

Extrapolating from this frequency of diagnosis and based on the estimated 2017 population of the United States of 325,719,178 (9), a rough estimate for the number of patients who are diagnosed with ME or CFS in the U.S. is 1.7 million to 3.4 million.

\section{Demographics of Diagnosed Population}

Detailed analysis of gender distribution by age for ME or CFS diagnosed individuals (no duplicates) within the three studied population sets are shown in Figures 2-4 and Tables 6, 7. Totals for the gender distribution are slightly smaller because gender information was not available for every individual. Results are normalized for each decile.

\section{Demographics of Diagnosed Population for the Main Dataset (Non-continuous Enrollment)}

Figure 2 and Table 6 show the gender distribution by age for individuals diagnosed with ME or CFS for the population of individuals who were enrolled at any time during the period 


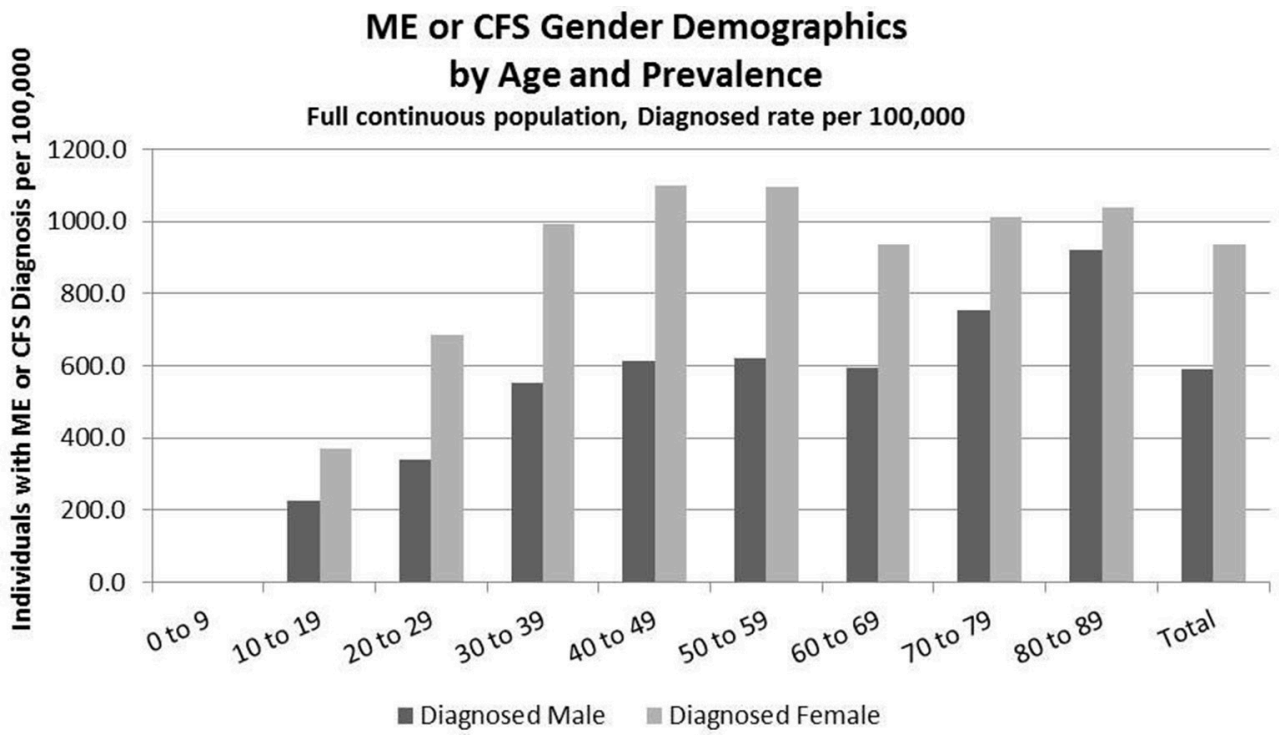

FIGURE 3 | ME or CFS gender demographics by age and prevalence (Subset 1, continuous enrollment from 2011 to 2016 ).

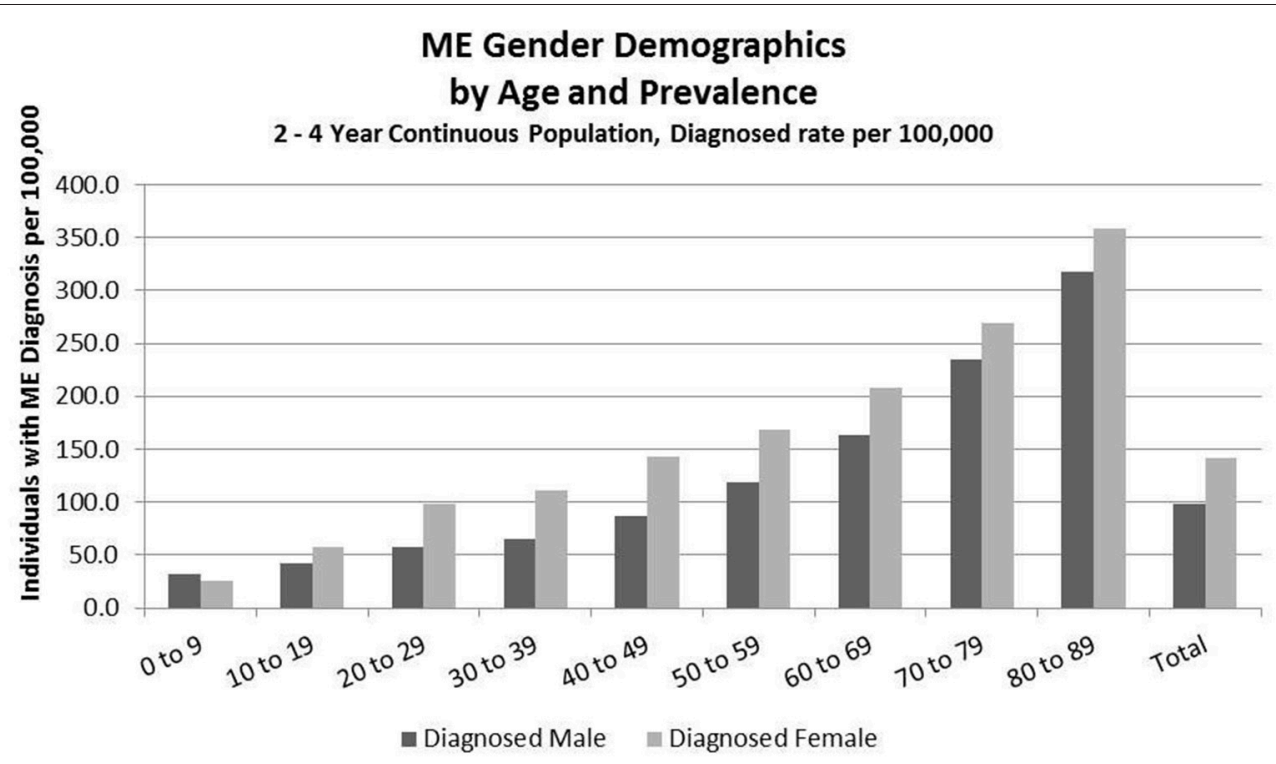

FIGURE 4 | ME gender demographics by age and prevalence (Subset 2, continuous enrollment from 2 to 4 years).

2011-2016, and the gender distribution by age and prevalence for these same individuals.

Of the 49,570,369 individuals enrolled during the period 2011-2016 for whom we have gender information, 258,702 (or $519 / 100,000$ ) had a code for diagnosis of either CFS or ME. The relative risk for females being diagnosed with ME or CFS compared to males was 1.238 (95\% CI: 1.235-1.242).

In the youngest age group, 0-9, boys outnumber girls; relative risk for females being diagnosed with ME or CFS compared to males in this age group was 0.922 (95\% CI: 0.874-0.970).

\section{Demographics of Diagnosed Population, Subset 1, Continuous Enrollment From 2011 to 2016}

Figure 3 and Table 7 show the gender distribution by age for individuals diagnosed with ME or CFS for the population of individuals who were continuously enrolled in their insurance for the entire period 2011-2016 (Subset 1) and the gender distribution by age and prevalence for these same individuals. In this group there were no diagnosed individuals younger than 10 . 
TABLE 6 | ME or CFS gender demographics by age and prevalence vs. reference population in the main dataset (Main dataset: non-continuous enrollment).

\begin{tabular}{|c|c|c|c|c|c|c|c|c|c|c|c|c|}
\hline \multirow[b]{2}{*}{ Age range } & \multicolumn{3}{|c|}{$\begin{array}{l}\text { Diagnosed-ME/CFS } \\
\text { (count/100,000) }\end{array}$} & \multirow{2}{*}{$\begin{array}{c}\text { F: M ratio } \\
\text { F: M }\end{array}$} & \multicolumn{2}{|c|}{$\begin{array}{l}\text { Diagnosed-ME/CFS } \\
\text { (\% normalized) }\end{array}$} & \multicolumn{3}{|c|}{$\begin{array}{c}\text { Diagnosed-ME/CFS } \\
\text { (count) }\end{array}$} & \multicolumn{3}{|c|}{ Data set population } \\
\hline & M & $\mathbf{F}$ & Total & & M & $\mathbf{F}$ & $\mathbf{M}$ & $\mathbf{F}$ & Total & $\mathbf{M}$ & $\mathbf{F}$ & Total \\
\hline 0 to 9 & 40.0 & 34.3 & 37.2 & $0.86: 1$ & $53.83 \%$ & $46.17 \%$ & 978 & 796 & 1,774 & $2,446,920$ & $2,321,941$ & $4,768,861$ \\
\hline 10 to 19 & 148.8 & 261.8 & 204.8 & 1.76: 1 & $36.24 \%$ & $63.76 \%$ & 4,023 & 6,934 & 10,957 & $2,702,876$ & $2,648,306$ & $5,351,182$ \\
\hline 20 to 29 & 259.5 & 478.1 & 379.3 & 1.84: 1 & $35.18 \%$ & $64.82 \%$ & 7,725 & 17,245 & 24,970 & $2,976,670$ & $3,606,744$ & $6,583,414$ \\
\hline 30 to 39 & 365.8 & 681.9 & 539.0 & 1.86: 1 & $34.91 \%$ & $65.09 \%$ & 11,679 & 26,390 & 38,069 & $3,192,640$ & $3,869,894$ & $7,062,534$ \\
\hline 40 to 49 & 482.1 & 909.1 & 708.4 & 1.89: 1 & $34.65 \%$ & $65.35 \%$ & 15,264 & 32,472 & 47,736 & $3,166,430$ & $3,571,767$ & $6,738,197$ \\
\hline 50 to 59 & 510.4 & 879.5 & 705.2 & 1.72: 1 & $36.72 \%$ & $63.28 \%$ & 16,865 & 32,459 & 49,324 & $3,304,031$ & $3,690,758$ & $6,994,789$ \\
\hline 60 to 69 & 499.6 & 739.6 & 628.1 & 1.48: 1 & $40.31 \%$ & $59.69 \%$ & 14,433 & 24,610 & 39,043 & $2,889,185$ & $3,327,290$ & $6,216,475$ \\
\hline 70 to 79 & 623.7 & 822.8 & 731.3 & 1.32: 1 & $43.12 \%$ & $56.88 \%$ & 9,976 & 15,479 & 25,455 & $1,599,520$ & 1,881,168 & $3,480,688$ \\
\hline 80 to 89 & 814.2 & 958.0 & 900.3 & 1.18: 1 & $45.94 \%$ & $54.06 \%$ & 7,766 & 13,608 & 21,374 & 953,817 & $1,420,412$ & $2,374,229$ \\
\hline Total & 381.8 & 645.4 & 521.9 & 1.69: 1 & $37.17 \%$ & $62.83 \%$ & 88,709 & 169,993 & 258,702 & 23,232,089 & $26,338,280$ & $49,570,369$ \\
\hline
\end{tabular}

TABLE 7 | ME or CFS gender demographics by age and prevalence vs. reference population (Subset 1: continuous enrollment 2011-2016).

\begin{tabular}{|c|c|c|c|c|c|c|c|c|c|c|c|c|}
\hline \multirow[b]{2}{*}{ Age range } & \multicolumn{3}{|c|}{$\begin{array}{l}\text { Diagnosed-ME/CFS } \\
\text { (count/100,000) }\end{array}$} & \multirow{2}{*}{$\begin{array}{c}\text { F: M ratio } \\
\text { F: M }\end{array}$} & \multicolumn{2}{|c|}{$\begin{array}{l}\text { Diagnosed-ME/CFS } \\
\text { (\% normalized) }\end{array}$} & \multicolumn{3}{|c|}{$\begin{array}{c}\text { Diagnosed-ME/CFS } \\
\text { (count) }\end{array}$} & \multicolumn{3}{|c|}{ Data set population } \\
\hline & $\mathbf{M}$ & $\mathbf{F}$ & Total & & $\mathbf{M}$ & $\mathbf{F}$ & $\mathbf{M}$ & $\mathbf{F}$ & Total & $\mathbf{M}$ & $\mathbf{F}$ & Total \\
\hline $0-9$ & 0.0 & 0.0 & 0.0 & & $0.00 \%$ & $0.00 \%$ & 0 & 0 & 0 & 4 & 5 & 9 \\
\hline 10 to 19 & 179.7 & 288.7 & 233.1 & 1.61: 1 & $38.36 \%$ & $61.64 \%$ & 242 & 374 & 616 & 134,694 & 129,563 & 264,257 \\
\hline $20-29$ & 297.3 & 584.0 & 443.8 & 1.96: 1 & $33.74 \%$ & $66.26 \%$ & 405 & 831 & 1,236 & 136,205 & 142,303 & 278,508 \\
\hline 30-39 & 481.6 & 881.7 & 695.9 & 1.83: 1 & $35.33 \%$ & $64.67 \%$ & 270 & 570 & 840 & 56,062 & 64,646 & 120,708 \\
\hline $40-49$ & 515.3 & 956.9 & 749.2 & 1.86: 1 & $35.00 \%$ & $65.00 \%$ & 780 & 1,631 & 2,411 & 151,354 & 170,449 & 321,803 \\
\hline 50-59 & 530.7 & 947.4 & 749.4 & 1.79: 1 & $35.91 \%$ & $64.09 \%$ & 1,155 & 2,277 & 3,432 & 217,625 & 240,347 & 457,972 \\
\hline $60-69$ & 527.7 & 819.9 & 682.5 & 1.55: 1 & $39.16 \%$ & $60.84 \%$ & 1,073 & 1,877 & 2,950 & 203,345 & 228,921 & 432,266 \\
\hline 70-79 & 640.2 & 848.8 & 752.9 & 1.33: 1 & $43.00 \%$ & $57.00 \%$ & 695 & 1,082 & 1,777 & 108,557 & 127,478 & 236,035 \\
\hline 80-89 & 791.5 & 909.7 & 862.8 & 1.15: 1 & $46.52 \%$ & $53.48 \%$ & 1,385 & 2,420 & 3,805 & 174,985 & 266,008 & 440,993 \\
\hline Total & 507.7 & 807.6 & 668.6 & 1.59: 1 & $38.60 \%$ & $61.40 \%$ & 6,005 & 11,062 & 17,067 & $1,182,831$ & 1,369,720 & $2,552,551$ \\
\hline
\end{tabular}

Of the 2,552,551 individuals continuously enrolled for the entire period 2011-2016 for whom we have gender information, 17,067 $(669 / 100,000)$ have a code for diagnosis of either CFS or ME; relative risk for females being diagnosed with ME or CFS compared to males was 1.210 (95\% CI: $1.196-1.223)$.

\section{Demographics of Diagnosed Population, Subset 2, Continuous Enrollment 2 to 4 Years}

Figure 4 and Table 8 show the gender distribution by age for individuals enrolled for a period of from 2 to 4 years and having a diagnosis code of ME in any diagnosis field in the claim, and the gender distribution by age and prevalence for these same individuals. The overall prevalence of a diagnosis of ME only (no CFS diagnosis) in the cohort continuously enrolled for 2 to 4 years is $121 / 100,000$. The relative risk for females being diagnosed with ME compared to males was 1.178 (95\% CI: 1.162$1.194)$. In the youngest age group, $0-9$, boys outnumber girls once again.

\section{Validation of Clinical Diagnostic Criteria}

In developing the list of appropriate symptom codes (Appendix 4 in Supplementary Material) it became apparent that existing codes do not fully identify symptoms that specifically describe ME/CFS. Most importantly, there is no symptom code specifically for post-exertional malaise, a core symptom, and codes for various types of fatigue do not match well with the description of another of the core symptoms, i.e., a substantial level of impairment in the ability to engage in pre-illness activities accompanied by fatigue.

Use of symptom codes relating to fatigue, sleep abnormalities, cognitive impairment and orthostatic intolerance, and without requiring codes possibly representing post-exertional malaise from consideration, resulted in a very small number of individuals who were diagnosed with ME or CFS. The vast majority of individuals who had a diagnosis code of ME or CFS did not appear in this symptomatic cohort (Figure 5).

\section{Machine Learning}

We were unable to create a model for ME and CFS together that could be trained and tuned to a sensitivity accuracy of 
TABLE 8 | ME gender demographics by age and prevalence vs. reference population (Subset 2, continuous enrollment 2 to 4 years).

\begin{tabular}{|c|c|c|c|c|c|c|c|c|c|c|c|c|}
\hline \multirow[b]{2}{*}{ Age range } & \multicolumn{3}{|c|}{$\begin{array}{l}\text { Diagnosed-ME } \\
\text { (count/100,000) }\end{array}$} & \multirow{2}{*}{$\begin{array}{c}\text { F: M ratio } \\
\text { F: M }\end{array}$} & \multicolumn{2}{|c|}{$\begin{array}{l}\text { Diagnosed-ME } \\
\text { (\% normalized) }\end{array}$} & \multicolumn{3}{|c|}{$\begin{array}{l}\text { Diagnosed-ME } \\
\text { (count) }\end{array}$} & \multicolumn{3}{|c|}{ Data set population } \\
\hline & $\mathbf{M}$ & $\mathbf{F}$ & Total & & $\mathbf{M}$ & $\mathbf{F}$ & $\mathbf{M}$ & $\mathbf{F}$ & Total & $\mathbf{M}$ & $\mathbf{F}$ & Total \\
\hline $0-9$ & 32.7 & 25.9 & 29.4 & 0.79: 1 & $55.81 \%$ & $44.19 \%$ & 207 & 157 & 364 & 633,270 & 606,551 & $1,239,821$ \\
\hline $10-19$ & 42.0 & 58.1 & 50.0 & 1.38: 1 & $41.97 \%$ & $58.03 \%$ & 307 & 412 & 719 & 730,324 & 708,729 & $1,439,053$ \\
\hline 20-29 & 57.4 & 97.8 & 77.5 & 1.70: 1 & $37.00 \%$ & $63.00 \%$ & 462 & 778 & 1,240 & 804,286 & 795,311 & $1,599,597$ \\
\hline 30-39 & 65.8 & 111.1 & 88.6 & 1.69: 1 & $37.20 \%$ & $62.80 \%$ & 545 & 932 & 1,477 & 827,930 & 838,616 & $1,666,546$ \\
\hline $40-49$ & 86.9 & 142.5 & 114.7 & 1.64: 1 & $37.89 \%$ & $62.11 \%$ & 668 & 1,099 & 1,767 & 768,545 & 771,386 & $1,539,931$ \\
\hline $50-59$ & 118.5 & 168.9 & 144.1 & 1.43: 1 & $41.22 \%$ & $58.78 \%$ & 903 & 1,335 & 2,238 & 762,241 & 790,364 & $1,552,605$ \\
\hline $60-69$ & 164.0 & 207.6 & 187.0 & 1.27: 1 & $44.13 \%$ & $55.87 \%$ & 1,090 & 1,540 & 2,630 & 664,725 & 741,738 & $1,406,463$ \\
\hline 70-79 & 234.3 & 268.7 & 253.1 & 1.15: 1 & $46.58 \%$ & $53.42 \%$ & 824 & 1,135 & 1,959 & 351,709 & 422,440 & 774,149 \\
\hline 80-89 & 317.9 & 358.1 & 342.5 & 1.13: 1 & $47.02 \%$ & $52.98 \%$ & 621 & 1,099 & 1,720 & 195,363 & 306,871 & 502,234 \\
\hline Total & 98.1 & 141.9 & 120.4 & 1.45: 1 & $40.87 \%$ & $59.13 \%$ & 5,627 & 8,487 & 14,114 & 5,738,393 & $5,982,006$ & $11,720,399$ \\
\hline
\end{tabular}

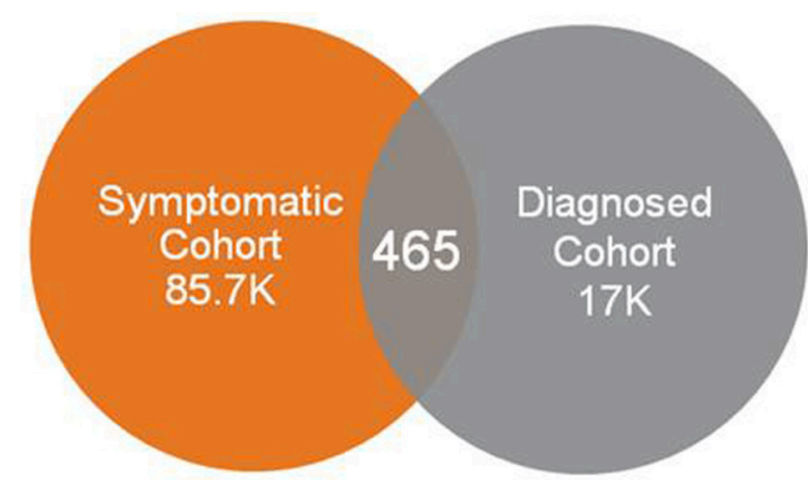

FIGURE 5 | Overlap of individuals with some symptoms of ME/CFS vs. those diagnosed.

much better than $50 \%$. This indicated that there was insufficient correlation between the input data (features) and outcome (diagnosis of ME or CFS) for the algorithm to make a useful prediction.

After failing to have the model resolve when including common symptom data along (CFS diagnosis), we refocused on diagnosis of ME (diagnosis presumed to include assessment of impaired function and the presence of PEM as core symptoms). The ME model was able to be trained and tuned successfully to achieve sensitivity of 0.738 (95\% CI: $0.721-0.754)$ and specificity of 0.823 (95\% CI: $0.823-0.823$ ) with the threshold set at 0.6 . The threshold signifies that the model will identify an individual as having ME if they have a risk score greater than or equal to $60 \%$.

Based on the machine learning predictive model, the projected prevalence of ME in our continuously insured population was $857 / 100,000$, calculated from the number of individuals predicted by the model to have an ME diagnosis when the model was evaluated using $99 \%$ probability $(3,989)$ and dividing by the total number of individuals in the dataset $(465,193)$. This methodology was used to capture individuals who are undiagnosed, but are most likely to be living with an ME-like illness. The gender distribution, normalized to size of population by gender, was 1.38:1 (58\% female and $42 \%$ male).

The top predictive features (those with the highest weights) in the model, which included both ICD and CPT codes submitted to insurance, are listed in Table 9.

\section{Costs}

Table 10 shows the average annual medical costs paid by insurance and the patient by year for individuals diagnosed with ME, as well as those diagnosed with lupus or multiple sclerosis, vs. those in the reference population. Costs used were the standard allowed payment (contracted rate) for all provider services which may have ultimately been paid by either the insurer or related patient responsibility associated with the claim such as patient co-payment or deductible, if any.

The average annual medical cost per individual diagnosed with $\mathrm{ME}$ in our dataset was $\$ 30,860$, while the average annual medical cost per individual in the general population in the database was $\$ 7,760$. For comparison, the average annual cost in our dataset for lupus patients was $\$ 20,160$ and for multiple sclerosis patients, $\$ 21,660$.

The costs varied by year, but on average, ME patients had medical costs that were three to four times greater than those in the general population, and $\sim 50 \%$ higher than either lupus or multiple sclerosis patients.

\section{DISCUSSION}

Prevalence of ME/CFS has been difficult to estimate due to a number of factors including lack of specific diagnostic tests, multiple case definitions, different methodologies, and confusion about coding. This study offers a new approach to this problem, using a large dataset of insurance claims to examine various characteristics of the group of patients for whom health care providers have given a diagnosis code for CFS or ME. We used a variety of data analysis techniques similar to those used in commercial research, which provide a range of estimates, and compare our results to other methods which have been used to estimate prevalence. 
TABLE 9 | Top predictive features for ME machine learning model.

\begin{tabular}{|c|c|c|}
\hline Score & Feature & Description \\
\hline 0.105990 & age & \\
\hline 0.017413 & gender & \\
\hline 0.016377 & icd_R53 & Malaise and fatigue \\
\hline 0.014899 & cpt_00175 & $\begin{array}{l}\text { Qualitative_or_Semiquantitative_ } \\
\text { Immunoassays }\end{array}$ \\
\hline 0.014763 & icd_N39 & Other disorders of urinary system \\
\hline 0.014508 & icd_E55 & Vitamin D deficiency \\
\hline 0.014083 & cpt_00123 & $\begin{array}{l}\text { Diagnostic_Radiology_(Diagnostic_Imaging)_ } \\
\text { Procedures_of_the_Head_and_Neck }\end{array}$ \\
\hline 0.013081 & cpt_00124 & $\begin{array}{l}\text { Diagnostic_Radiology_(Diagnostic_Imaging)_ } \\
\text { Procedures_of_the_Chest }\end{array}$ \\
\hline 0.012911 & icd_R07 & Pain in throat and chest \\
\hline 0.012452 & cpt_00128 & $\begin{array}{l}\text { Diagnostic_Radiology_(Diagnostic_Imaging)_ } \\
\text { Procedures_of_the_Abdomen }\end{array}$ \\
\hline 0.011824 & icd_R51 & Headache \\
\hline 0.011824 & cpt_00217 & Cardiography_Procedures \\
\hline 0.011178 & cpt_00168 & Urinalysis_Procedures \\
\hline 0.010957 & icd_R06 & Abnormalities of breathing \\
\hline 0.010499 & icd_RO0 & Abnormalities of heart beat \\
\hline 0.010465 & icd_R94 & Abnormal results of function studies \\
\hline 0.010431 & cpt_00289 & Subsequent_Hospital_Care_Services \\
\hline 0.010074 & icd_R50 & Fever of other and unknown origin \\
\hline 0.009819 & icd_D64 & Other anemias \\
\hline 0.009751 & icd_E03 & Other hypothyroidism \\
\hline 0.009429 & cpt_00367 & Temporary_National_Codes_(Non-Medicare) \\
\hline 0.009378 & cpt_00220 & Echocardiography_Procedures \\
\hline 0.008987 & icd_K59 & Other functional intestinal disorders \\
\hline 0.008885 & cpt_00350 & $\begin{array}{l}\text { Ambulance_and_Other_Transport_Services_ } \\
\text { and_Support }\end{array}$ \\
\hline 0.008596 & cpt_00174 & Hematology_and_Coagulation_Procedures \\
\hline 0.008392 & icd_Z51 & Encounter for other aftercare and medical care \\
\hline 0.008307 & icd_M62 & Other disorders of muscle \\
\hline 0.00739 & icd_R79 & Other abnormal findings of blood chemistry \\
\hline 0.007339 & cpt_00160 & Diagnostic_Nuclear_Medicine_Procedures \\
\hline 0.007203 & icd_R26 & Abnormalities of gait and mobility \\
\hline
\end{tabular}

\section{Coding and Diagnosis Considerations}

The diagnostic codes for CFS (in ICD-10-CM) and for ME (in both ICD-10-CM and ICD-9-CM) are not exclusive to these diseases and can include other conditions, which introduces an unknown degree of uncertainty into any prevalence estimates based on these diagnostic codes (see Appendix 1 in Supplementary Material, Interrelationships of ICD codes used for Chronic Fatigue Syndrome (CFS) and Myalgic Encephalomyelitis (ME) in the U.S. as of October, 2018).

A proposal to change the coding for ME, CFS, and related conditions was made at the September 12, 2018 meeting of the National Center for Health Statistics which addresses this ambiguity (10). If this proposal is approved, ME, CFS, Systemic Exercise Intolerance Disease (SEID, the new term recommended by the Institute of Medicine report in 2015), and postviral fatigue
TABLE 10 | Average yearly medical costs for diagnosed vs. reference population.

\begin{tabular}{lcccc}
\hline Year & $\begin{array}{c}\text { General } \\
\text { population }\end{array}$ & ME & Lupus & MS \\
\hline 2016 & $\$ 8,500$ & $\$ 30,600$ & $\$ 22,600$ & $\$ 23,220$ \\
2015 & $\$ 7,800$ & $\$ 32,400$ & $\$ 21,100$ & $\$ 22,090$ \\
2014 & $\$ 7,500$ & $\$ 31,300$ & $\$ 20,100$ & $\$ 21,050$ \\
2013 & $\$ 7,700$ & $\$ 34,300$ & $\$ 20,100$ & $\$ 22,780$ \\
2012 & $\$ 7,300$ & $\$ 25,700$ & $\$ 16,900$ & $\$ 19,160$ \\
Average & $\$ 7,760$ & $\$ 30,860$ & $\$ 20,160$ & $\$ 21,660$
\end{tabular}

syndrome will have separate and distinct codes beginning in October 2019.

For better tracking of this disease, two options could be considered. In the short term, and if the new proposal is not approved, providers who diagnose ME/CFS could use the ICD10-CM code of G93.3 for ME/CFS and not use R53.82 (Chronic Fatigue, unspecified). Ultimately, if the proposed changes are approved, researchers could use the specific codes for the conditions they are tracking.

Diagnosis may vary depending on the case definition or diagnostic criteria used by the provider. Furthermore, there is considerable ongoing investigation on the effect of using different case definitions on the diagnosis of ME and or CFS (11), and this affects whether it is legitimate to use an umbrella term to describe the two conditions (12). While we refer to ME/CFS in this study, our analysis is based on diagnosis of ME and CFS separately, as identified by the specific diagnostic codes for each, although there is no way to know how each medical provider makes a diagnosis and assigned a code.

\section{Prevalence of ME/CFS Based on Frequency of Diagnosis in an Insurance Claims Database}

This is the first study to determine the frequency of ME/CFS diagnosis using insurance claims data for a large number of individuals. Prevalence estimates for chronic fatigue syndrome in the U.S. have been as low as 235/100,000 $(373 / 100,000$ in women and $83 / 100,000$ in men) (13) and as high as $2540 / 100,000$ (14). Our prevalence estimates ranged from $519 / 100,000$ to $1,038 / 100,000 \quad(0.52 \%$ to $1.03 \%$ ), which fall between those expected from largescale health surveys and smaller scale community-based studies.

Our highest prevalence estimate for ME/CFS, 1,038/100,000 or $1.04 \%$, was found in the group most representative of the insured population-those continuously enrolled for 2 to 4 years-and with the broadest reach: it includes all individuals with ME or CFS as either a primary or a secondary diagnosis, and all claims for these individuals. This estimate can be compared with health surveys conducted in Canada and in some states in the U.S. Recent CDC prevalence estimates of ME/CFS from the Behavioral Risk Factor Surveillance System (BRFSS) (lifetime 1.6\%; current 1.2\%) (15) were similar to 
Canadian Community Health Survey 2003 (1.3\%) 2010 (1.4\%), and $2014(1.4 \%)$ data (16-18). These survey studies do not verify that the specific diagnosis code has been entered in the patient's medical record. They only indicate that the patient is reporting having been given this diagnosis by the health care provider.

Our lowest prevalence estimate for ME/CFS, 519/100,000 or $0.52 \%$, was calculated using all claims from individuals who have $\mathrm{ME}$ or CFS as the primary diagnosis and with no restriction on the length of enrollment. This group could therefore have an unknown number of individuals with only one miscellaneous claim, thereby diluting the sample. Nevertheless, the prevalence of ME/CFS in this group is higher than predicted by communitybased studies which verified the diagnosis with a medical examination and verifying symptoms using an accepted ME or CFS case definition (e.g., Jason et al., (19), 0.42\%; 7, 0.24\%; $10,0.2 \%)$.

Our intermediate prevalence estimate has no direct comparisons with previously published results. The prevalence of ME/CFS in the group continuously enrolled for the full 7 years and with $\mathrm{ME}$ or CFS as the primary diagnosis, the most restrictive group, is $669 / 100,000$ or $0.67 \%$. Note that this is somewhat higher than the $519 / 100,000$ calculated from the non-continuously enrolled population. The group of individuals continuously enrolled in the same health insurance for a long period of time may include a higher proportion of sicker people than the other groups, but we did not assess this.

Using the diagnosis of $\mathrm{ME}$ only, prevalence in the group continuously enrolled for 2 to 4 years is $121 / 100,000$, or $0.12 \%$. This lower prevalence of ME compared with ME/CFS would be expected, as the case definitions for ME are much less wellknown by medical providers than CFS. Nevertheless, our sample included more than 14,000 individuals with this diagnosis, which is quite large compared with most studies which examine the characteristics of this group.

Using claims data alone, it is not possible to determine what criteria health care providers are using to make a diagnosis of either ME or CFS. Likewise, a provider might tell a patient they have ME/CFS without the specific diagnostic code being entered into the patient's record.

The prevalence of ME/CFS could be overestimated if providers or medical coders are using the CFS diagnosis code to identify a "CFS-like" illness or condition, without reference to any case definition, or simply "chronic fatigue."

The prevalence of ME/CFS could be underestimated if providers or medical coders (a) use a different diagnosis that is less specific (e.g., 780.79, Other Malaise and Fatigue, R53.81 Other Malaise or R53.83 Other Fatigue); (b) do not put ME or CFS diagnosis into the record due to not wanting to expose their patients to a perceived stigma of the disease (1), due to not wanting to provide a discouraging diagnosis when there is no cure, or due to knowing that some appropriate treatments might not be covered under that diagnosis; and (c) if providers are unaware of the diagnosis of ME/CFS, since diagnosis and management of ME/CFS is not taught in most medical schools $(20,21)$.
It has been reported (8) that $84-91 \%$ of patients with ME/CFS are undiagnosed. However, this study is now 14 years old, and so may not reflect the increased awareness of ME/CFS in recent years, which could result in a higher rate of diagnosis. The earlier case definitions used in previous studies require the diagnosis to be one of exclusion, resulting in less likelihood of diagnosis than with more recent clinical diagnostic criteria published by the Institute of Medicine (1).

\section{Age, Gender, and Prevalence in the Diagnosed Population}

This study also shows a lower ratio of females to males diagnosed with ME/CFS than is generally reported. While many studies show a much higher percentage of females (as high as $80 \%$ female $-7 ; 9)$ at least one health survey (17) shows a lower percentage of $65 \%$ female. For ME/CFS, our studies show an average of between 60 and $65 \%$ female across age groups, except in the youngest group, 0-9 years, where boys outnumber girls. For ME only, the percentage of females is lower, $60 \%$, and again for the youngest group, boys outnumber girls.

While our numbers are based on diagnostic codes in the medical record and reflect actual clinical practice, there is no information about what criteria the providers used to assign these codes, or if they evaluate men differently than women. Nevertheless, the higher than expected number of males with this diagnosis is interesting, and the possible reasons for this need more study.

\section{Prevalence in Children and Adolescents}

There is little published data on prevalence of ME/CFS in children and adolescents. One community-based study reports a prevalence for adolescents (aged 13 to 17) of 181 per 100,000 or $0.181 \%$ (22). Our main dataset shows a prevalence of $37.2 / 100,000$ in children $0-9$ years, and 204.5/100,000 in ages 10-19 (Table 6).

\section{Validation of Clinical Diagnostic Criteria}

One goal of the study was to determine whether the presence of specific symptom codes within administrative medical claims data could identify individuals for whom a diagnosis of ME/CFS should be considered. Lack of specific codes for two of five core symptoms required for a diagnosis of ME/CFS using the IOM criteria made it impossible to identify individuals for whom this diagnosis should be considered from administrative claims data, or to validate that individuals diagnosed with ME/CFS had documented evidence of the required core symptoms in their claims data.

\section{Prevalence Based on Machine Learning}

Based on the 2017 population of the U.S. noted earlier, the predicted prevalence rate based on our model of $857 / 100,000$ translates to up to 2.8 million people with ME/CFS in the U.S. This number is somewhat larger than other published estimates of 836,000 to 2.5 million Americans (19) and is significant because it is predicted based on characteristics drawn from those diagnosed with ME only, not including those with a diagnosis of CFS only. 
The machine learning technique is a useful way to compensate for the lack of specific symptom codes which might otherwise be used to predict or identify undiagnosed patients. It uses a weighted analysis of a large number of "features" (over 500) derived from a known group (in this case, individuals already diagnosed with ME) to identify individuals with a similar combination of factors. The model can be "tuned" to a desired balance of specificity and sensitivity. Our model performed reasonably well at a threshold of 0.6 (sensitivity 0.82336 and specificity 0.73787 ). If specific symptom codes for $\mathrm{ME}$ were available the model could be improved. To predict the prevalence of ME from our dataset we used a probability threshold of 0.99 . Using the $99 \%$ probability cut-off is a conservative approach, but provides a reasonable estimate.

The inability to train the machine learning model when CFS diagnoses were included indicates that the population of individuals diagnosed with CFS is too heterogeneous for this method. In contrast, individuals diagnosed with ME were a more homogenous population for which this approach was more effective.

The CFS diagnosis code, in the signs and symptoms section of the ICD, is perhaps being used incorrectly to indicate the symptom of chronic fatigue, which is characteristic of many different underlying conditions, or a "CFS-like" illness which may lack some of the defining features of Chronic Fatigue Syndrome. However, patients coded with ME, which has clinical information identical to Chronic Fatigue Syndrome in ICD$10-\mathrm{CM}$ and is placed in a disease chapter, were significantly, and usefully, more homogeneous. This supports our supposition that clinicians using the ME code are more familiar with the disease than clinicians using the CFS code, and thus may be specifically diagnosing ME/CFS, not using the diagnosis code to cover unspecified chronic fatigue or a "CFS-like" illness.

These results show that the predicted prevalence rate of $857 / 100,000$ based on the machine learning model is not unreasonable for $\mathrm{ME} / \mathrm{CFS}$, including the symptom of postexertional malaise. This estimate suggests that ME/CFS is not a rare disease, but in fact a relatively common one, and offers a new benchmark for future studies.

\section{Costs}

Direct medical costs are important to insurers, who need to deliver good medical care in a cost-effective way, and to patients, who must pay both insurance premiums and out-of-pocket for co-payments, deductibles and treatments that are uninsured.

Direct medical costs for caring for ME/CFS patients are significantly higher than for the general population. Specific components contributing to increased costs (hospitalization, specialist visits, diagnostic tests, presence of other chronic conditions, etc.) were not examined.

Many patients cite a long and costly journey to receiving an accurate diagnosis of ME/CFS (23). Further, once diagnosed, most patients struggle to find primary care providers who are knowledgeable about the condition and well versed in the best practices for managing the symptoms. These twin challenges in diagnosis and treatment are certainly contributors to added cost in the healthcare system.
Direct medical costs are only one component of the total disease costs; others include disability claims, health insurance premiums, and expenses not submitted to insurance such as alternative treatments, nutritional supplements, costs to the economy due to productivity loss, costs to the family for caretaking, and possibly early death. Previous studies have estimated the total annual cost to the economy from ME/CFS to be \$17-24 billion (2008 dollars) (6).

Patients with ME/CFS have a high level of disability. Despite high direct medical costs, these patients often have significant unmet health care needs (17) or forgo routine medical care (15). Health surveys have indicated that ME/CFS patients also tend to have more than one chronic condition (15). All these factors could combine and result in poorer quality of life for the patient and even higher medical costs in the future, as well as increasing the burden of illness.

The data from this study illustrate the high costs of the illness, and point to the potential for cost control if patients are diagnosed and provided with the most effective care. Good medical management also holds the promise of improving the experience of patients living with ME/CFS.

\section{Putting the Results in Context Prevalence}

The estimated prevalence of ME/CFS in our study ranges from 519 to $1,038 / 100,000$, and falls between the rates estimated from community health studies and self-reported health surveys. Our study uses larger samples than previous studies, and two different methodologies. Our studies show a range of gender distribution, with the lowest ratio of female to male occurring in the youngest age group, 0-9 years, where boys outnumber girls, and in groups diagnosed with ME only.

Table 11 illustrates the spectrum of prevalence studies which use a variety of techniques. Comparing these studies shows the range of prevalence and gender distribution. Bolded entries are from this study.

Generally accepted gender ratios for ME/CFS in the community are as high as $3: 1$ or $4: 1$ female to male F $(75-80 \%$ female). Our data indicate that the actual rate of diagnosis is much less skewed based on gender, though still more commonly diagnosed in women, with a range of $60-65 \%$ female.

\section{Disease Burden}

The World Health Organization has pioneered the use of the Disability Adjusted Life Year (DALY) as a single measure of disease burden in a population (25) and importantly, it includes a measure of the degree of disability from the disease. Using the DALY measure, ME/CFS has been estimated to have a higher total disease burden than multiple sclerosis, autism, or HIV/AIDS (26).

Lupus and multiple sclerosis (MS) are two diseases which are better known than ME/CFS and often compared to it. Although they have different etiologies they have some similar characteristics and symptoms. Both significantly affect quality of life, may take some time to diagnose, affect more women than men, present with some of the same symptoms, and like ME/CFS are often diagnosed late and/or inaccurately initially. 
TABLE 11 | Comparison of prevalence rates.

\begin{tabular}{|c|c|c|c|c|}
\hline Source & $\begin{array}{l}\text { Population } \\
\text { size }\end{array}$ & $\begin{array}{l}\text { Prevalence } \\
\text { per } 100,000\end{array}$ & $\%$ Female & Method \\
\hline Diagnosed with ME (subset 2, continuous enrollment 2-4 years) & $11.7 \mathrm{M}$ & 121 & $60.1 \%$ & Insurance Claim Data \\
\hline Nacul et al. (24), (ME/CFS, U.K.) & 143,000 & 200 & $51.0 \%$ & Community Health Study \\
\hline Reyes et al. (13) (ME/CFS, Wichita, KS) & 90,316 & 240 & $81.8 \%$ & Community Health Study \\
\hline Jason et al. (19) (ME/CFS, U.S.) & 18,675 & 420 & $71.9 \%$ & Community Health Study \\
\hline Diagnosed with ME or CFS (main dataset, non-continuous enrollment) & $50 \mathrm{M}$ & 519 & $65.7 \%$ & Insurance Claim Data \\
\hline Diagnosed with ME or CFS (subset 1, continuous enrollment 2011-2016) & $2.5 \mathrm{M}$ & 669 & $64.7 \%$ & Insurance Claim Data \\
\hline Projected prevalence of ME using machine learning & $2.7 \mathrm{M}$ & 857 & $57.9 \%$ & Machine Learning Predictive Model \\
\hline Diagnosed with ME or CFS (subset 2, continuous enrollment 2 to 4 years) & $11.7 \mathrm{M}$ & 1038 & $65.0 \%$ & Insurance Claim Data \\
\hline $\begin{array}{l}\text { National ME/FM Action Network (17) and ME Association of Ontario (16) } \\
\text { (Canadian Community Health Surveys) }\end{array}$ & 65,000 & 1,400 & $63.4 \%$ & Survey \\
\hline Lin et al. (15) (BRFSS survey, ME/CFS, several states) & 54,695 & 1,600 & $80.0 \%$ & Survey \\
\hline
\end{tabular}

TABLE 12 | Comparison of several factors relating to ME/CFS, lupus and multiple sclerosis.

\begin{tabular}{|c|c|c|c|c|c|}
\hline Disease & $\begin{array}{l}\text { \# Patients based on } \\
\text { est. } 2017 \text { U.S. } \\
\text { population }\end{array}$ & Prevalence & $\begin{array}{l}\text { Burden of illness } \\
\text { (DALY-disability adjusted } \\
\text { life years) }\end{array}$ & $\begin{array}{l}\text { Average annual } \\
\text { medical cost }\end{array}$ & $\begin{array}{l}\text { NIH research spending } \\
2017 \text { (NIH categorical } \\
\text { spending, 2017) }\end{array}$ \\
\hline ME/CFS & $1,726,000-3,746,000$ & $\begin{array}{l}519-1,038 / 100,000 \\
0.52-1.04 \%\end{array}$ & $714000(26)$ & $\$ 30,860$ & $\$ 15 \mathrm{MM}$ \\
\hline Lupus & 785,000 & $\begin{array}{l}241 / 100,0000.241 \% \\
(27)\end{array}$ & No data available & $\$ 20,160$ & $\$ 109 \mathrm{MM}$ \\
\hline Multiple Sclerosis & 486,000 & $\begin{array}{l}149 / 100,000 \\
0.15 \%(28)\end{array}$ & $\begin{array}{l}300200(26)-284171(\mathrm{NIH}, \\
\text { disease burden 2015) }\end{array}$ & $\$ 21,000$ & $\$ 111 \mathrm{MM}$ \\
\hline Reference population & & & & $\$ 7,760$ & \\
\hline
\end{tabular}

Table 12 compares the estimated prevalence and number of patients in the United States, the burden of illness, and average annual medical cost, and NIH research spending for ME/CFS, lupus, and multiple sclerosis $(29,30)$. As shown in Table 12, $\mathrm{ME} / \mathrm{CFS}$ affects more than double the number of persons in the U.S. than lupus and four times as many as MS. The prevalence of lupus is less than half that of ME/CFS, and the prevalence of multiple sclerosis, in a comparable study of commerciallyinsured patients, is less than one-third of the prevalence of $\mathrm{ME} / \mathrm{CFS}$ as found in our study.

The burden of illness for ME/CFS is more than double that of MS (26), and medical costs for ME/CFS in this study are double those for either Lupus or MS and four times higher than for the general insured (reference) population.

An additional point of comparison is the amount spent on research for these diseases by the National Institutes of Health. Looking at these comparisons for prevalence, burden of illness and annual medical cost, and the amount of NIH funding for these three similar diseases, ME/CFS, lupus, and multiple sclerosis, it is evident that research on ME/CFS is grossly underfunded (\$15 vs. \$109-111 MM), a point also made by Dimmock et al. (26).

\section{Limitations of This Type of Study}

This study, based on claims data including Medicare and commercial insurance, does not assess the prevalence in
Medicaid recipients or the uninsured, two groups in which the prevalence of ME/CFS might be higher, as indicated by community-based studies (19). Furthermore, the financial impact of disability from ME/CFS may lead to Medicaid eligibility, thus removing some ME/CFS patients from the commercially insured population.

Since we were not able to validate ME/CFS diagnosis using codes for some (but not all) of the core symptoms, our machine learning model results must be considered preliminary.

ICD codes used for ME/CFS as of 2018 are inexact and may be applied to individuals with other conditions (see Appendix 1 in Supplementary Material). This introduces an unknown degree of uncertainty to the estimates of prevalence of ME/CFS in this study. In doing this study, we necessarily made a number of assumptions which are stated in the Methods section and are also discussed above. The results might have been different if different assumptions were used. Since this data is from U.S. insurance claims and reflects the practices of U.S. health care providers, these results may not be valid for other countries.

\section{Implications for the Future and Next Steps}

The authors recommend use of the ME diagnosis code (G93.3) rather than CFS (R53.82), which defaults to "Chronic fatigue, unspecified," for better tracking where symptoms warrant.

The authors also recommend the creation of new symptom codes for post-exertional malaise and substantial impairment in 
activity levels accompanied by profound fatigue, two of the core symptoms of ME/CFS.

\section{In Summary}

This study is the first to use a large medical claims database to study the characteristics of a large group of individuals who have been diagnosed with ME or CFS and to explore the potential of mining this type of data. This study used a base data set of 50 million individuals tracked over 6 years. The next largest study referenced had a sample size of 90,316, all located in a single municipality.

While the percentage of women diagnosed with ME/CFS is higher than the percentage of men, ME/CFS is not a "women's disease." Thirty-five to forty percent of diagnosed patients are men.

It is not possible at this time to use symptom codes in medical claims data to identify individuals for whom a diagnosis of ME/CFS might be considered. Introducing new symptom codes for two of the required symptoms identified in the study published by the Institute of Medicine (1) should be considered.

Patients diagnosed with CFS may represent a more heterogeneous group than those diagnosed with $\mathrm{ME}$; this study makes no conclusions about accuracy of diagnosis or quality of care given to ME/CFS patients by providers.

Annual direct medical costs for ME/CFS patients are three to four times higher than average of the reference population and fifty percent higher than for multiple sclerosis or lupus, diseases with similar characteristics.

This study is a "snapshot" and could be repeated in future years for comparison. It would also be interesting to look in more detail at the diagnosis of conditions which are co-morbid with $\mathrm{ME} / \mathrm{CFS}$, such as migraine headaches or orthostatic intolerance.

\section{REFERENCES}

1. Institute of Medicine. Beyond Myalgic Encephalomyelitis/Chronic Fatigue Syndrome: Redefining an Illness. Washington, DC: The National Academies Press. (2015). Available online at: http://www.nationalacademies.org/hmd/ Reports/2015/ME-CFS.aspx (Accessed September 15, 2018).

2. Smith MEB, Nelson HD, Haney E, Pappas M, Daeges M, Wasson $\mathrm{N}$, et al. Diagnosis and Treatment of Myalgic Encephalomyelitis/Chronic Fatigue Syndrome. Agency for Healthcare Research and Quality, Evidence Reports/Technology Assessments (2014).

3. Chronic Fatigue Syndrome Advisory Committee. CFSAC Recommendations: Recommendations and Rationale from Working Group on IOM and P2P Reports-PDF (2015). Available online at: https://www.hhs.gov/ash/advisorycommittees/cfsac/recommendations/2015-08-18/index.html and https:// www.hhs.gov/sites/default/files/advcomcfs/recommendations/2015-08-1819-recommendations.pdf (Accessed September 15, 2018).

4. Marshall R, Paul L, Wood L. The search for pain relief in people with chronic fatigue syndrome: a descriptive study. Physiother Theor Pract. (2011) 27: 373-83. doi: 10.3109/09593985.2010.502554

5. National Institutes of Health. State of the Knowledge Workshop. Myalgic Encephalomyelitis/Chronic Fatigue Syndrome (ME/CFS) Research. Workshop report. Bethesda, MD: Office of Research on Women's Health, NIH, US Department of Health and Human Services (2011). Available online at: https://www.meassociation.org.uk/wp-content/uploads/2011/08/SoKWorkshop-Report-508-compliant-8-5-11.pdf (Accessed September 15, 2018).
These results show that a prevalence rate $857 / 100,000$ for $\mathrm{ME} / \mathrm{CFS}$ is not unreasonable; therefore, it is not a rare disease, but in fact a relatively common one.

Based on our results and analysis, ME/CFS should get more attention in research and provider communities, and warrants more education to providers (primary care, specialties, and allied health sciences) to improve the quality of health care and quality of life for affected individuals.

\section{AUTHOR CONTRIBUTIONS}

Research for this study was carried out by AV, EH, SA, and $\mathrm{DK}$, with direction and support from $\mathrm{DP}$ and JA. $\mathrm{LB}, \mathrm{AD}, \mathrm{CL}$, and $\mathrm{PR}$ provided clinical guidance. AD advised on statistics. The manuscript was written primarily by $\mathrm{AV}, \mathrm{DP}$, and $\mathrm{CP}$, with editorial suggestions from $\mathrm{LB}, \mathrm{AD}, \mathrm{CL}, \mathrm{PR}$, and JA.

\section{ACKNOWLEDGMENTS}

We are grateful to Optum Enterprise Analytics, Optum Technology, and UnitedHealth Group for making staff time available for this project, and to Mary Dimmock, Arthur Mirin, and Leah Williams for helpful comments. We are grateful for donations to the Massachusetts ME/CFS \& FM Association which funded the publication of this manuscript.

\section{SUPPLEMENTARY MATERIAL}

The Supplementary Material for this article can be found online at: https://www.frontiersin.org/articles/10.3389/fped. 2018.00412/full\#supplementary-material

6. Jason LA, Benton MC, Valentine L, Johnson A, Torres-Harding S. The economic impact of ME/CFS: Individual and societal costs. Dyn Med. (2008) 7:6. doi: 10.1186/1476-5918-7-6

7. Reynolds KJ, Vernon SD, Bouchery E, Reeves WC. The economic impact of chronic fatigue syndrome. Cost Effect Resour Alloc. (2004) 2:4. doi: 10.1186/1478-7547-2-4

8. Solomon L, Reeves WC. Factors influencing the diagnosis of chronic fatigue syndrome. Arch Intern Med. (2004) 164:2241-5. doi: 10.1001/archinte.164.20.2241

9. United States Census Bureau (2018). Population estimates. Available online at: https://www.census.gov/quickfacts/fact/table/US/PST045217

10. National Center for Health Statistics. ICD-10 Coordination and Maintenance Committee Meeting, Diagnosis Agenda Part 2 (2018). Available online at: https://www.cdc.gov/nchs/data/icd/Topic_packet_Sept_2018_part2.pdf (Accessed September 30, 2018).

11. Jason LA, Brown A, Clyne E, Bartgis L, Evans M, Brown M. Contrasting case definitions for chronic fatigue syndrome, myalgic encephalomyelitis/chronic fatigue syndrome and myalgic encephalomyelitis. Eval Health Prof. (2012) 35:280-304. doi: 10.1177/0163278711424281

12. Jason LA, Sunnquist M, Brown A, Evans M, Newton JL. Are myalgic encephalomyelitis and chronic fatigue syndrome different illnesses? A preliminary analysis. J Health Psychol. (2016) 1:3-15. doi: 10.1177/1359105313520335

13. Reyes M, Nisenbaum R, Hoaglin DC, Unger ER, Emmons C, Randall B, et al. Prevalence and incidence of chronic fatigue syndrome in Wichita, Kansas. Arch Intern Med. (2003) 163:1530-6. doi: 10.1001/archinte.163.13.1530 
14. Reeves WC, Jones JF, Maloney E, Heim C, Hoaglin DC, Boneva RS, et al. Prevalence of chronic fatigue syndrome in metropolitan, urban, and rural Georgia. Popul Health Metr.(2007) 5:5. doi: 10.1186/14787954-5-5

15. Lin JMS, Hayes K, Brimmer D. 9630: BRFSS State-Added Questions: Leveraging an Existing Surveillance System to Monitor Prevalence and Health Indictors for Myalgic Encephalomyelitis/Chronic Fatigue Syndrome (ME/CFS). In: 2018 CSTE Annual Conference, Council of State and Territorial Epidemiologists Palm Beach, FL. (2018). Available online at: https:// cste.confex.com/cste/2018/meetingapp.cgi/Paper/9630 (Accessed August 15, 2018).

16. ME Association of Ontario. THE QUANTITATIVE DATA: Environmental Sensitivities/Multiple Chemical Sensitivity (ES/MCS), Fibromyalgia (FM), Myalgic Encephalomyelitis/Chronic Fatigue Syndrome (ME/CFS). Appendix to The Ontario Centre Of Excellence in Environmental Health Business Case (2013). Available online at: http://meao.ca/files/Quantitative_Data_Report. pdf

17. National ME/FM Action Network. Myalgic Encephalomyelitis/Chronic Fatigue Syndrome and Fibromyalgia Findings from the 2014 Canadian Community Health Survey (2014). Available online at: https://www.mefmaction.com/docs/ CCHS_Stats_2014.pdf (Accessed August 4, 2018).

18. Rusu C, Gee ME, Lagacé C, Parlor M. Chronic fatigue syndrome and fibromyalgia in Canada: prevalence and associations with six health status indicators. Health Promot Chronic Dis Prev Can. (2015) 35:3-11.

19. Jason LA, Richman JA, Rademaker AW, Jordan KM, Plioplys AV, Taylor R, et al. A community-based study of chronic fatigue syndrome. Arch Intern Med. (1999) 159:2129-37.

20. Peterson TM, Peterson TW, Emerson S, Regalbuto E, Evans MA, Jason LA. Coverage of CFS within U.S. medical schools.Univers J Public Health (2013) 1: 177-9. doi: 10.13189/ujph.2013.010404

21. Jason LA, Paavola E, Porter N, Morello ML. Frequency and content analysis of chronic fatigue syndrome in medical text books. Austral J Primary Health (2010) 16:174-8. doi: 10.1071/py09023

22. Jordan KM, Huang CF, Jason LA, Richman J, Mears CJ, McCready W, et al. Prevalence of pediatric chronic fatigue syndrome in a community-based sample. J Chron Fatigue Syndr. (2006) 13:75-8. doi: 10.1300/J092v13n02_04

23. ProHealth. A Profile of ME/CFS Patients: How Many Years and How Many Doctors? (2008). Available online at: https://www.prohealth.com/library/aprofile- of-me-cfs-patients-how-many-years-and-how-many-doctors- 25211 (Accessed September 15, 2018).
24. Nacul LC, Lacerda EM, Pheby D, Campion P, Molokhia M, Fayyaz S, et al. Prevalence of myalgic encephalomyelitis/chronic fatigue syndrome (ME/CFS) in three regions of England: a repeated cross-sectional study in primary care. BMC Med. (2011) 9:91. doi: 10.1186/1741-7015-9-91

25. Murray CJ, Lopez AD, and Jamison DT. The global burden of disease in 1990: summary results, sensitivity analysis and future directions. Bull World Health Organ. (1994) 72:495-509.

26. Dimmock ME, Mirin AA, Jason LA. Estimating the disease burden of ME/CFS in the United States and its relation to research funding. J Med Ther. (2016) 1: 1-7. doi: 10.15761/JMT.1000102

27. Rees F, Doherty M, Grainge MJ, Lanyon P, Zhang W. The worldwide incidence and prevalence of systemic lupus erythematosus: a systematic review of epidemiological studies. Rheumatology (2017) 56:1945-61. doi: 10.1093/rheumatology/kex260

28. Dilokthornsakul P, Valuck RJ, Nair KV, Corboy JR, Allen RR, Campbell JD. Multiple sclerosis prevalence in the United States commercially insured population. Neurology (2016) 86:1014-21. doi: 10.1212/WNL.0000000000002469

29. National Institutes of Health. Report on NIH Funding vs. Global Burden of Disease (2015). Available online at: https://report.nih.gov/info_disease_ burden.aspx (Accessed August 25, 2018).

30. National Institutes of Health. Estimates of Funding for Various Research, Condition, and Disease Categories (RCDC) (2018). Available online at: https:// report.nih.gov/categorical_spending.aspx

Conflict of Interest Statement: AV, EH, DK, DP, and JA are employees of UnitedHealth Group. SA is a former employee of UnitedHealth Group.

The remaining authors declare that the research was conducted in the absence of any commercial or financial relationships that could be construed as a potential conflict of interest.

Copyright (C) 2019 Valdez, Hancock, Adebayo, Kiernicki, Proskauer, Attewell, Bateman, DeMaria, Lapp, Rowe and Proskauer. This is an open-access article distributed under the terms of the Creative Commons Attribution License (CC BY). The use, distribution or reproduction in other forums is permitted, provided the original author(s) and the copyright owner(s) are credited and that the original publication in this journal is cited, in accordance with accepted academic practice. No use, distribution or reproduction is permitted which does not comply with these terms. 